\title{
KLOE-2 Inner Tracker: the First Cylindrical GEM Detector
}

\author{
Erika De Lucia*† \\ Laboratori Nazionali di Frascati dell'INFN (IT) \\ E-mail: erika.delucia@lnf.infn.it
}

\begin{abstract}
KLOE-2 at the DAФNE $e^{+} e^{-}$collider is the main experiment of the INFN Laboratori Nazionali di Frascati (LNF) and is the first high-energy experiment using GEM technology with a cylindrical geometry, a novel detector developed at LNF exploiting kapton properties. Four concentric cylindrical triple-GEM detectors compose the Inner Tracker, inserted around the interaction region and before the inner wall of the pre-existing KLOE Drift Chamber, to improve the resolution on decay vertices close to the interaction point and reconstructed from low-momentum charged secondaries.

State-of-the-art solutions have been expressly developed or tuned for this project: single-mask GEM etching, now used also for several LHC experiments upgrades, multi-layer XV patterned readout circuit, PEEK spacer grid, GASTONE front-end board a custom 64-channel ASIC with digital output, and the Global Interface Board for data collection, with a configurable FPGA architecture and Gigabit Ethernet.
\end{abstract}

Alignment and calibration of a cylindrical GEM detector was never done before and represented one of the experiment's challenging activities. The first set of alignment and calibration parameters obtained with cosmic-ray muon data has been used to validate the integrated tracking and vertexing using both Inner Tracker and Drift Chamber information, exploiting the Kalman filter technique. Results obtained with $\phi \rightarrow \pi^{+} \pi^{-} \pi^{0}$ and $K_{S} \rightarrow \pi^{+} \pi^{-}$samples will be reported.

KLOE-2 is presently taking data with the aim of collecting more than $5 \mathrm{fb}^{-1}$ by March 2018.

The success of the cylindrical GEM technology developed by the KLOE- 2 collaboration paved the way to its usage in other high-energy physics experiments, among these is the BES III experiment at BEPC II.

The European Physical Society Conference on High Energy Physics

5-12 July, 2017

Venice

\footnotetext{
* Speaker.

${ }^{\dagger}$ For the KLOE-2 Collaboration.
} 


\section{Introduction}

KLOE-2 experiment represents the continuation of KLOE [1] at the DAФNE $e^{+} e^{-}$collider [2] with a new physics program [3] mainly focused on the study of $\mathrm{K}_{S}, \eta$ and $\eta^{\prime}$ decays as well as on kaon interferometry, tests of discrete symmetries, and search for new exotic particles as possible manifestation of the dark matter $[4,5]$. In particular, this new data taking campaign will allow CPT symmetry and quantum coherence tests using entangled neutral kaons to be performed with an unprecedented precision [6].

The general purpose original KLOE detector, composed by one of the biggest drift chamber (DC) ever built [7] surrounded by a lead-scintillating fiber Electromagnetic Calorimeter [8] among the best ones for energy and timing performance at low energies, undergone several upgrades to improve its discovery potential. These include state-of-the-art cylindrical GEM detector, the Inner Tracker, LET and HET taggers [9] for $\gamma \gamma$-fusion physics, and the CCALT and QCALT detectors [10], crystal and tile calorimeters positioned near the interaction point and along the beam-pipe respectively, to improve multi-photon detection in rare decays and background rejection power.

KLOE-2 data taking campaign started in November 2014, reached $3.7 \mathrm{fb}^{-1}$ integrated luminosity in June 2017 and is presently ongoing with the aim of collecting at least $5 \mathrm{fb}^{-1}$ by March 2018.

\section{The First Cylindrical GEM detector construction}

In order to improve its vertex reconstruction capabilities near the interaction region, KLOE-2 experiment is using GEM technology with a cylindrical geometry, a novel idea that was developed at LNF exploiting the kapton properties to build a transparent and compact tracking system [11]. This innovative tracking detector, the Inner Tracker (IT), has been inserted in the free space between the beam pipe and the DC inner wall, at $25 \mathrm{~cm}$ from interaction point (IP). The IT is the first ever built and operated cylindrical GEM detector, with a total material budget below $2 \%$ of radiation length, allowing to minimize the multiple scattering of low-momentum tracks and the probability of photon conversions.

Four concentric cylindrical triple-GEM detectors (CGEM) at distances from $130 \mathrm{~mm}$ to 205 mm compose the Inner Tracker. Each CGEM is a triple-GEM detector composed by five concentric cylindrical electrodes: the cathode, to set the drift field, 3 GEM foils for the electron multiplication and the anode, acting also as readout circuit.

Three years of R\&D demonstrated the feasibility of a cylindrical triple-GEM detector $[12,13$, 14] and included the tuning and development of a new manufacturing procedure of GEM foils of unprecedented size (up to $50 \times 100 \mathrm{~cm}^{2}$ ) with a single-mask electro-chemical etching of the microholes, produced with the TE-MPE-EM CERN group and followed and supported within the RD51 Collaboration [15]. Single-mask etching technique is presently used for several LHC experiments upgrades based on GEM detectors.

State-of-the-art multi-layer XV patterned anode readout circuit has been expressly developed with TE-MPE-EM CERN group: longitudinal X strips with $650 \mu \mathrm{m}$ pitch are interleaved, on the same substrate and at the same level, with pads connected through internal vias to form $\mathrm{V}$ strips at an angle within $25^{\circ} \div 27^{\circ}$ and with $600 \mu \mathrm{m}$ pitch, for a total of about $30,000 \mathrm{FEE}$ channels. Space 
coordinates are reconstructed coupling the anode readout to custom on- and off-detector electronics developed for KLOE-2: the GASTONE front-end, a 64-channel ASIC with digital output [16], and the General Interface Board (GIB) for data collection with a configurable FPGA architecture, Gigabit Ethernet and the readout driver (ROD) [17].

\section{Operation with collisions}

The operational parameters have been optimized as a function of the DAФNE $\mathrm{e}^{+} \mathrm{e}^{-}$beam currents and machine background conditions. Dedicated on-line procedures are used to monitor IT temperature, currents and voltages, together with occupancy and clustering performance [18] with DAФNE delivering collision data. The detector is operated with an $\mathrm{Ar}: \mathrm{iC}_{4} \mathrm{H}_{10}$ 90:10 gas mixture at a nominal effective gain of about $10^{4}$, with electric fields set at $1.5 / 3 / 3 / 6 \mathrm{kV} / \mathrm{cm}$ for drift/transfer1/transfer2/induction gaps and GEM1/GEM2/GEM3 voltages set at 280/280/270 V respectively. At the IT working point a satisfactory single-view efficiency of $\sim 92 \%$ and a twoview efficiency of $\sim 84 \%$ have been measured with cosmic-ray muon tracks reconstructed with DC information [19]. The two-view efficiency is confirmed with collision data using Bhabha scattering events [20].

\section{Alignment \& Calibration}

Alignment and calibration of a novel detector as the CGEM have been among the challenging activities of the KLOE-2 project. Two effects with 1-2 mm average corrections were observed and measured with planar XV prototypes during the $R \& D$ phase of the project[11]. The first effect is related to non-radial tracks crossing the detector and the second is due to the presence of the magnetic field. In order to evaluate the first set of alignment and calibration parameters, cosmic-ray muon data samples with and without magnetic field have been acquired and then Bhabha scattering events have been used as validation. DC excellent track reconstruction has been exploited.

The IT-DC residual distributions obtained with Bhabha scattering events and using the first alignment and calibration exhibit average widths along the $\mathrm{x}$-axis $\operatorname{Res}(\mathrm{x}) \sim 400 \mu \mathrm{m}$ for all layers, to be compared with the starting value of $1.5 \mathrm{~mm}$ without any alignment and calibration. The residuals are all centered around zero within $50 \mu \mathrm{m}$ [20]. These results are very close to expectations and will improve with presently ongoing refinements of the alignment and calibration procedure. Preliminary results obtained with the second set of alignment and calibration parameters, from the refined procedure, show $\sim 100 \mu \mathrm{m}$ reduction on $\operatorname{Res}(\mathrm{x})$ from both cosmic-ray muon data without and with magnetic field.

\section{Integrated tracking performance}

IT clusters are included in the reconstruction, starting from DC hits and track parameters, by using the Kalman filter technique and then updating track parameters. The vertex finding algorithm has been updated as well accounting for the additional IT information. Bhabha scattering events and decays close to the IP seen by both DC and IT detectors have been used for benchmark studies 
of the newly IT+DC integrated tracking and vertexing algorithms. Preliminary results have been obtained using the first set of alignment and calibration parameters.

Due to the negligible beam size contribution along the vertical direction, tens of $\mu \mathrm{m}$, the distribution of the y coordinate YV of the reconstructed vertex position of $\phi \rightarrow \pi^{+} \pi^{-} \pi^{0}$ decays represents a good figure of merit of the vertex resolution close to the IP. Fig.1 left shows the improvement in the YV distribution of $\phi \rightarrow \pi^{+} \pi^{-} \pi^{0}$ decays, obtained using the integrated tracking. Fitting the distribution with a double Gaussian function, the $3 \mathrm{~mm}$ standard deviation of the narrow component obtained with DC-only reconstruction is reduced to about $2 \mathrm{~mm}$ with the integrated reconstruction.

Similar results have been obtained with $K_{S} \rightarrow \pi^{+} \pi^{-}$decays (Fig.1 right) in which the YV distribution is the convolution of beam size, vertex resolution and $K_{S}$ lifetime. The total $\sigma_{\mathrm{DC}} \sim$ $1 \mathrm{~cm}$ of the double Gaussian fit to the YV distribution obtained with DC-only reconstruction is reduced to $\sigma_{\mathrm{IT}+\mathrm{DC}} \sim 0.7 \mathrm{~cm}$ using the integrated reconstruction.
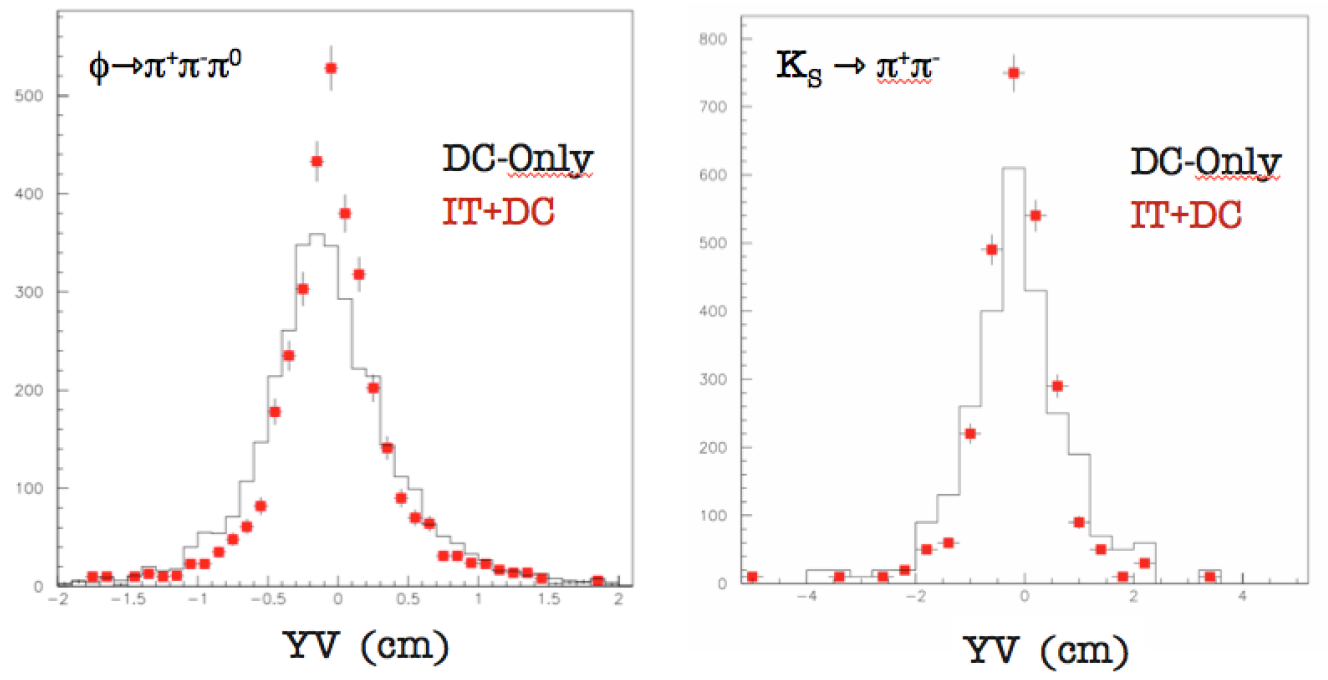

Figure 1: The distribution of the y coordinate of the reconstructed vertex position of $\phi \rightarrow \pi^{+} \pi^{-} \pi^{0}$ (Left) and $K_{S} \rightarrow \pi^{+} \pi^{-}$(Right) decays: comparison between DC-only (solid line) and integrated IT+DC reconstruction (points).

Preliminary results on the fit to the $K_{S}$ proper time distribution in $\tau_{S}$ units, obtained with the first integrated tracking version, show a total resolution improvement from $1.4 \tau_{S}$ DC-only to $1 \tau_{S}$ already using the first set of IT alignment and calibration parameters. Improvement are expected with the refined alignment and calibration and further integrated tracking optimization.

\section{Conclusions}

The KLOE detector has been upgraded with several new sub-detectors for the new data taking campaign within the KLOE-2 project. The KLOE-2 Inner Tracker is the first CGEM detector operated, aligned and calibrated in a high-energy physics experiment. Good results from the integrated tracking and vertexing obtained with $\phi \rightarrow \pi^{+} \pi^{-} \pi^{0}$ and $K_{S} \rightarrow \pi^{+} \pi^{-}$decays have been shown already with the first set of alignment and calibration parameters. Improvements are 
expected with the refined procedure. KLOE-2 integrated luminosity is $3.7 \mathrm{fb}^{-1}$ as of June 2017 and the goal is to acquire at least $5 \mathrm{fb}^{-1}$ by the end of March 2018.

\section{References}

[1] F. Bossi, E. De Lucia, J. L. Franzini, S. Miscetti, M. Palutan and KLOE Collaboration, Nuovo Cimento, 30 (2008) 10.

[2] A. Gallo (Frascati) et al., Conf.Proc. C060626 (2006) 604-606, SLAC-PUB-12093, M. Zobov et al., Phys. Rev. Lett. 104, 174801 (2010), C. Milardi et al., JINST, 7, 2012, T03002.

[3] G. Amelino Camelia et al., Physics with the KLOE-2 experiment at the upgraded DAФNE, Eur. Phys. J. C 68619 (2010).

[4] A. Kupsc for the KLOE-2 Collaboration, KLOE-2 results on hadron physics, these proceedings

[5] G. Mandaglio for the KLOE-2 Collaboration, KLOE/KLOE-2 results and perspectives on dark force search, these proceedings

[6] A. Di Domenico for the KLOE-2 Collaboration, Test of discrete symmetries with neutral kaons at $K L O E-2$, these proceedings

[7] M. Adinolfi et al., Nucl. Inst. \& Meth. A 488 (2002) 51.

[8] M. Adinolfi et al., Nucl. Inst. \& Meth. A 482 (2002) 51.

[9] Babusci D. et al., Acta Phys. Pol. B, 46 (2015) 81.

[10] Happacher F. and Martini M., Acta Phys. Pol. B, 46 (2015) 87.

[11] F. Archilli et al., KLOE-2 Collaboration, Technical design report of the inner tracker for the KLOE-2 experiment, arXiv:1002.2572v1 and LNF-10/3(P) INFN-LNF, Frascati,2010.

[12] G. Bencivenni et al., A Time Domain Reflectometer with 100 ps precision implemented in a cost-effective FPGA for the test of the KLOE-2 Inner Tracker readout anodes, Nucl. Inst. \& Meth. A 698 (2013) 185.

[13] A. Balla et al.,The cylindrical GEM detector for the KLOE-2 Inner Tracker, JINST 9 C01014 (2014).

[14] A. Balla et al., Status of the cylindrical-GEM project for the KLOE-2 inner tracker, Nucl. Inst. \& Meth. A 628 (2011) 18.

[15] M. Alfonsi et al., Activity of CERN and LNF groups on large area GEM detectors, Nucl. Inst. \& Meth. A 617 (2010) 151-154; presented at the $11^{\text {th }}$ Pisa Meeting on Advanced Detector, May 24-30, 2009, La biodola - Isola d'Elba (Italy).

[16] A. Balla et al., GASTONE64: A new front-end ASIC for the cylindrical GEM Inner Tracker of KLOE-2 experiment at DAФNE, Nucl. Inst. \& Meth. A 721 (2013) 523.

[17] P. Branchini et al., Front-end DAQ strategy and implementation for the KLOE-2 experiment, JINST 8 T04004 (2013).

[18] A. Di Cicco, G. Morello, Commissioning of the Inner Tracker of the KLOE-2 experiment, Acta Phys. Polon. B 46 (2015) 73.

[19] A. Balla et al., The KLOE-2 Inner Tracker: Detector commissioning and operation, Nucl. Inst. \& Meth. A 845 (2017) 266.

[20] G. Bencivenni et al., The cylindrical GEM detector of the KLOE-2 experiment, JINST 12 C07016 (2017). 